\title{
Orbital Muscle Entrapment
}

National Cancer Institute

\section{Source}

National Cancer Institute. Orbital Muscle Entrapment. NCI Thesaurus. Code C118752.

The binding of one or more ocular muscles by orbital bone pieces, resulting in decreased eye mobility. 